AL-AZHAR UNIVERSITY

BULLETIN OF THE FACULTY OF

LANGUAGES \& TRANSLATION

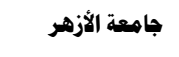

هبلة كلية اللغات والترجمة

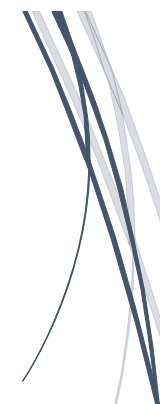

\title{
La frase condizionale-ipotetica in italiano e in arabo
}

Studio contrastivo

Dr. Amal Kamal Abdulhafiz 


\title{
The conditional-hypothetical sentence in Italian and Arabic Contrastive study
}

Amal Kamal Abdulhafiz

Department of Italian Language, Faculty of Arts, Helwan University, Cairo, Egypt.

\begin{abstract}
:
This research deals with the different times of the condition and the values of the condition clause in general and the values of the default condition clause beginning with "se" in the Italian language. The research aims to highlight the grammatical properties of the condition clause in Italian and Arabic and study translation problems. The method used is the deductive analytical approach deductive. The subject elements were studied by extracting examples from linguistics books and through a literary story to study translation problems. The research consists of two consecutive parts: The first part is the theoretical part devoted to the study of the times of the condition and its use accompanied by examples and the study of the properties of the sentence of the condition of the condition and the sentence of the condition of the condition and its types and the study of the condition in the present tense and the past time and its use in independent sentences and non-independent sentences and formulas of the simple and complex condition and its use in disciplinary demand To express a desire and give advice, and to express doubt and suspicion. The condition is used in the past tense to express an event that could have happened in the past and did not happen and will not happen in the present and future and expresses the future in the past and that it can be used in the rigid source form.
\end{abstract}

Keywords: Condition Sentence, Arabic, Italian, Problems, Translation.

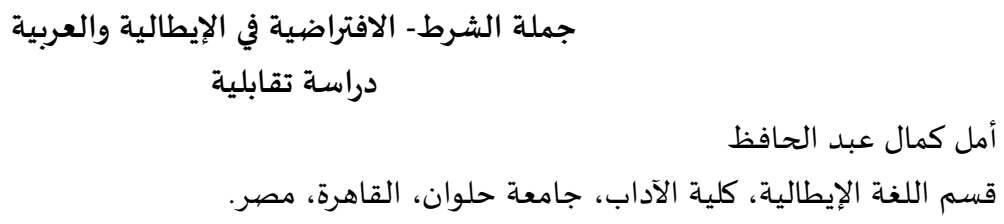




\section{L'introduzione}

Prima di tutto è necessario chiarire che la ricerca tratta il condizionale della lingua italiana, i valori della frase condizionale in generale e poi quelli della frase condizionale-ipotetica introdotta da" $s e^{\prime \prime}$. L'obiettivo della ricerca è illustrare i casi particolari in cui viene usato il condizionale e la frase condizionale e rilevare le caratteristiche grammaticali della frase condizionale in italiano e in arabo e studiare i problemi della traduzione.

Il metodo usato è quello contrastivo- analitico con l'approccio deduttivo, quindi, la ricerca è composta di due parti successive:la prima è la parte teorica che è dedicata all'uso del condizionale e alle caratteristiche della frase-condizionale e della frase-conseguenza, del periodo ipotetico. La seconda è la parte pratica che è dedicata ai punti di corrispondenza accompagnati dai problemi di traduzione attraverso alcune frasi ricavate dalla storia "l'inseguimento" ${ }^{(1)}$ di Italo Calvino per esaminare anche i problemi della traduzione.

Nella lingua italiana scritta o parlata l'uso del periodo ipotetico considerato più comune perché lo permette a esprimere la certezza, la possibilità, l'impossibilità.

\section{Il condizionale:}

prima parlerò in breve del condizionale e poi della frase condizionale ipotetica:

patota $^{(2)}$ dice che il condizionale si presenta l'azione o il modo di essere come eventuali-ipotetici; e ciò è, come realizzabili, nel presente o nel passato, ma subordinatamente a determinati condizioni che possono essere espressi o sottintesi. Tali condizioni sono per lo più indipendenti dalla volontà di chi parla o scrive e possono risultare: o già ben definiti ed esistenti o supponibili oppure suggeriti da opportunità di adattamento comportamentale a specifici aspetti situazionali. Mediante il condizionale manifesta l'attegiamento mentale o psicologico del consapevole distacco o del sospeso possibilismo o della cauta esitazione.

Per esemplificare: apodosi: vorrei parlare

(protasi: se ha un po' di tempo).

\footnotetext{
${ }^{1}$ Italo Calvino, L'inseguimento, traduzione del prof., Moheb Saad, in Narrativa Italiana Contemporanea, vol. II, Istituto Italiano Di Cultura, II Cairo 1988.

${ }^{2} \mathrm{Cfr}$.Giuseppe Patota, Grammatica di riferimento della lingua italiana per stranieri, "Società Dante Alighieri", le Monnier, Firenze 2003, p.145-146.
} 


\section{L'uso del condizionale:}

Il condizionale può stare invece dell'indicativo nelle preghiere, nelle interrogazioni, e quando si vuol togliere asprezza alla frase escludendo ogni idea di comando, di pretesa, di superbia, specie in frasi di cortesia: Vorrei una sigaretta! Andresti anche tu? Direi che sarebbe meglio; Mi faresti un favore?

Anche per esprimere dubbio e meraviglia: una donna? E chi sarebbe? sarebbe lei, veramente?!

-Il condizionale indica fatti, azioni, modi di essere in cui prevale l'aspetto di eventualità, subordinata a una condizione (di qui il nome): me ne anderei (se potessi)

Carlo Rossi sarebbe stato messo in prigione. (come a dire: se è vera la notizia che ho sentito, Carlo Rossi...)

Dardano e Trifone $e^{3}$ sottolineano che

- il condizionale è per eccellenza un modo che si adopera nella proposizione principale per esprimere un'azione, un avvenimento o uno stato che si realizza o può realizzarsi in dipendenza dall'avverarsi di condizioni espresse nella proposizione subordinata o anche sottaciute e sottintese: sarei andato anche io con voi, se non avessi avuto da terminare il mio lavoro, Oh di che cuor con voi mi resterei cioè "se potessi".

- Il parlante può presentare il fatto espresso dal verbo in diversi modi, ciascuno dei quali indica un diverso punto di vista, un diverso atteggiamento psicologico, un diverso rapporto comunicativo con chi ascolta: certezza, possibilità, desiderio, comando ecc.

Talvolta, poi, l'uso di un determinato modo può dipendere anche da ragioni stilistiche, da una scelta di "registro" o di livello linguistico: così, per esempio nelle subordinate rette da verbi di giudizio l'indicativo ( $\mathrm{mi}$ pare che ha ragione) corrisponde a un livello d'espressione più popolare rispetto al congiuntivo (mi pare che abbia ragione)

\footnotetext{
${ }^{3} \mathrm{Cfr}$., Maurizio Dardano e Pietro Trifone, Grammatica italiana con nozioni di linguistica, Zanichelli, Bologna,1995, pp.305, 311, 351, 450.
} 
3- Nella lingua dell'informazione giornalistica e televisiva, per presentare una notizia come possibile o probabile, ma non certa:il segretario dell'ONU sardebbe già partito alla volta di Buenos Aires.

4- Per esprimere un dubbio (in particolare, coi verbi potere, dovere, volere): Dove potremmo andare a cena stasera, Che vorresti dire

5- Per esprimere un desiderio o un augurio: come sarebbe bello fare una gita a Venezia!

In quasi tutti i casi appena presentati, il condizionale sembra indicare la conseguenza di una condizione non espressa, ma sottintesa: vorrei una pizza margherita (sottinteso: se lei non ha nulla in contrario),

\section{La sintassi della frase condizionale}

-Il condizionale nelle seguenti dipendenti

Silvestrini e Bura ${ }^{4}$ dicono che il condizionale si può trovare nelle seguenti dipendenti: 1. oggettive (dopo una enunciativa): penso che sarebbe meglio andare a casa.

2.interrogative indirette: gli studenti si chiedono che cosa potrebbero fare.

3.causali: mi dispiace tanto, perché avrei darvi una brutta notizia.

4.consecutive: ero così stanco che mi sarei buttato per terra a dormire.

5.avversative: ora sei qui da me, ma dovresti essere altrove.

6.comparative: era più bello di quanto si sarebbe potuto immaginar.

7.relative: sono state indicate molte persone che avverbbero avuto a che fare con i traditori.

8.incindentali: la vita direi l'esistenza, e fatta anche di tante delusioni.

\section{-Il periodo ipotetico}

Dardano e Trifone $e^{5}$ ribadiscono che il periodo ipotetico è formato da due proposizioni in stretta correlazione tra loro, di cui esprime la condizione necessaria

${ }^{4} \mathrm{Cfr}$. Marcello Silvestrini e Claudio Bura, L'italiano e L' Italia, Roma, pp.147-148.

${ }^{5} \mathrm{Cfr}$. Maurizio Dardano e Pietro Trifone, La lingua italiana, Zanichelli, Bologna 1985 pp.303- 304. 
per l'avverarsi di quanto è affermato nell'altra: se comincia a parlare ,non la finisce più.

La proposizione subordinata condizionale (quella che esprime la condizione) viene chiamata PROTASI (dal greco pro`tasis 'premessa'); l'apodosi (dal latino greco apo`dosis 'conseguenza') è appunto la 'conseguenza ' che si dichiara nella reggente : se continuerà a piovere :protasi (prop. Condizionale), resteremo a casa: apodosi (prop.reggente)

Con la congiunzione "se", il modo del verbo è l'indicativo per esprimere un'ipotesi reale (se comincia a parlare, non la finisce più); è il congiuntivo per esprimere un'ipotesi possibile (se dovessi andare via, passerei prima a salutare) o irreale (andrei più spesso all'estero, se conoscessi le lingue). Quando la proposizione condizionale è introdotta da una congiunzione diversa da "se" o da una locuzione congiuntiva, il modo della protasi è sempre il congiuntivo. Infatti, tutte le congiunzioni , eccetto se, e tutte le locuzioni congiuntive esprimono solo ipotesi possibili o irreali. Con "se" possiamo avere l'indicativo in entrambe (realtà) o il congiuntivo nella prima e il condizionale nella seconda (possibilità, irrealtà)

\section{-La frase condizionale nella forma implicita}

Nella forma implicita, le proposizioni condizionali possono essere rappresentate da un gerundio, da un participio passato o dall'infinito del verbo: applicando, potresti rendere molto di più (= se ti applicassi). Sviluppata meglio, sarebbe un'ottima idea (=se fosse sviluppata meglio). A vederlo, non penseresti che è ricco (=se lo vedessi...)

reggente; presente / futuro *penso / penserò, passato *pensavo /pensai/ho pensato Subordinata; contemproraneità: che tu finiresti anteriorità: che tu avresti finito posteriorità: che tu finiresti / che tu potresti finire posteriorità: che tu avresti finito L'apodosi può essere una proposizione principale (se partecipassi alla gara vinceresti, se partecipassi alla gara).

La congiunzione condizionale più comune è SE: si usano anche: qualora, purché', ove, e le locuzioni posto che ,ammesso che ,nell'eventualità che , nell'ipotesi che ecc...

\section{La funzione del condizionale:}

La funzione tipica del condizionale (presente o passato) è quella di esprimere la conseguenza (apodosi di una premessa condizionante (protasi) 
espressa o sottintesa nell'ambito del periodo ipotetico della possibilità e della irrealtà. È ciò, come si è detto, in proposizione tanto principale che subordinata: -la faresti felice (principale) se andassi a casa sua (possibilità) - l'avresti fatta (passato) felice se fossi andato a casa tua (irrealtà nel passato).

-per Natale andrei volentieri in montagna. (protasi sottintesa)

\section{La conseguenza con il condizionale:}

-come si esprime una conseguenza all'interno collegata a una frase condizione introdotta da SE, qualora ecc. -il modo in cui si esprime una conseguenza segnata da un elemento -condizione che la precede, e che può essere un aggettivo (tanto,tale,simile ), un avverbio (tanto ,così , talmente), un aggettivo +un avverbio (così tanto, sì,talmente ), un aggettivo + un avverbio (così tanto, così simile ); due avverbi (così tanto, talmente tanto ) insieme di parola (a tal punto, in modo tale ,in modo, in maniera tale ). Questo tipo di conseguenza è espresso da una frase (detta consecutiva) formata da: - che+ verbo allindicativo (o al congiuntivo o al condizionale): ero così stanco che mi sono addormentato subito "tanto ho fatto , preso un spavento tale che alla fine hanno invitato anche lui "

-Al posto dell'indicativo si adopera il congiuntivo quando la conseguenza è anche uno scopo: -"gli parlerò in maniera tale che mi capisca " -"gli hanno ingessato la gamba in modo che possa camminare "-Invece, al posto dell'indicativo si adopera il condizionale quando la conseguenza è possibile a patto che si realizzi una condizione sottintesa : -Venezia è così bella che ci vivrei (condizionale sottintesa :se potessi) . -quello è uno talmente ricco che potrebbe comparare tutto l'albergo (condizione sottintesa:se volesse). l'elemento-condizione e la congiunzione " che" possono anche fondersi in un'unica espressione: cosicché (o così che), tanto che , di modo che , al punto che ecc..".da" +verbo all'infinito : possibile che sia talmente ingenuo da non aver capito niente ? _la frase che esprime la conseguenza può avere questa forma solo se: -il soggetto dell'infinito è lo stesso della frase precedente: "Monica Bellucci è così affascinante da incontrare il pubblico in ogni cosa che fa " -il soggetto dell'infinto è generico e imprecisato: "fa così caldo da sentirsi male " _l'elemento-condizione e la preposizione "da" possono anche fondersi in un'unica espressione : tanto da, così da, in modo da . A questo elenco va aggiunta l'espressione al punto di: mi sono commosso al punto di piangere -di una conseguenza si può esprimere non solo l'effettiva realizzazione, ma anche la sola possibilità o impossibilità di realizzazione . In questo caso l'elemento condizione è un avverbio come troppo, poco troppo poco ,abbastanza, e la possibilità e l'impossibilità della conseguenza è espressa da una frase formata da : - 
perchè +verbo al congiuntivo : federico è abbastanza grande perchè gli si possa parlare,la casa ha un prezzo troppo alto perchè possiamo parlare con i soldi che abbiamo -per o da + verbo all'infinito (presente o passato) : Valeria è abbastanza grande per uscire da sola,

ho comprato una lavatrice troppo difficile da usare.

\section{La frase condizionale:}

Patota $^{6}$ aggiunge che- la frase condizionale (o protasi del periodo ipotetico), è introdotta dalla congiunzione SE, mentre la frase -conseguenza (o apodosi del periodo ipotetico) non ha introduttori ;puo` essere aperta (ma raramente ) della parola allora: se dicessi ,(allora)sbaglieresti.

-la posizione della frase -condizione e della frase-conseguenza non è fissa; generalmente la frase-condizione precede la frase-conseguenza, ma quest'ordine non e’ affatto obbigatorio, e può aversi quello contrario : -se potessi , (frasecondizione) cambierei casa (frase-conseguenza). -cambierei casa, (frase conseguenza) se potessi (frase-condizione).

\section{-Tipi di ipotesi:}

Patota $^{c}$ divide l'ipotesi in tre tipi dicendo che

Un'ipotesi può essere presentata come certa, come possibile o come irreale

Tipo di ipotesi Frase-condizionale Frase-conseguenza

Certa Se io ho fatto Hai sbagliato Possibile possibile Se dicessi Sbaglieresti

Irreale Se fossi (stato) un animale Vorrei (avrei voluto) essere un cane

\section{-Tempi e modi dell'ipotesi reale:}

un'ipotesi reale puo`avere tutti i tempi dell'indicativo sia nella frase condizione sia nella frase-conseguenza (inoltre, nella, frase-conseguenza possiamo trovare l'imperativo ): se le cose stanno cosi ,non importa'

(indicativo) se lo hai fatto arrabbiare, chiedigli scusa (imperativo) tempi e modi dell'ipotesi possibile o irreale: a) se l'ipotesi o irreale è riferita al presente, troveremo il congiuntivo imperfetto nella frase -condizione e il condizionale

${ }^{6} \mathrm{Cfr}$., Giuseppe Patota, op. cit., pp.379- 380 . 
presente nella frase -conseguenza : se mi offrissero (congiuntivo imperfetto )un buon lavoro , accetterei (condizionale presente) subito". b) se l'ipotesi possibile o irreale è riferita al passato, troveremo il congiuntivo trapassato nella frasecondizione e il condizionale passato nella frase-conseguenza : se avessi studiato (congiuntivo

trapassato) di più, agli esami saresti andato (condizionale passato) meglio

-nel caso però che i fatti indicati nella frase-conseguenza avvengono nel presente, si usa il condizionale presente al posto del condizionale passato:se avessi ascoltato (congiuntivo trapassato) i miei consigli, oggi vivresti (condizionale presente) meglio.

- Riassumendo:

Ipotesi

Certa

\section{Conseguenza}

Condizione

o irreale (presente) Se + congiuntivo imperfetto, condizionale presente

Possibile o irreale (passato) Se+congiuntivo(trapassato)

Condizione passato

Indicativo imperfetto nell'ipotesi irreale -spesso, per esprimere un'ipotesi irreale ,nell'italiano parlato ,al congiuntivo e del condizionale ,si adopera l'indicativo imperfetto :se mi avvertivate prima, la piazza la portavo Io l'ipotesi irreale "mista" -l'indicativo imperfetto può trovarsi anche solo nella frasecondizione ,insieme al condizionale passato nella frase-conseguenza : se mi avvertivate prima ,la pizza l'avveri portata Io ; oppure solo nella frase- conseguenza ,insieme al congiuntivo trapassato nella frase-condizione : se mi aveste avvertito prima,la pizza la portavo Io . Entrambe queste forme rientrano in quello che in sintassi è indicato come il "periodo ipotetico misto". Bisogna sottolineare che l'uso dell'indicativo imperfetto nella frase-condizione dell'ipotesi irreale è normale nell'italiano parlato ma è da evitare nell'italiano scritto. in cui è preferibile usare il congiuntivo nella frase-condizione e il condizionale nella frase-conseguenza ed evitare il congiuntivo nella frase- conseguenza. frase-condizione non introdotta da SE come si è già accennato, la frase -condizione è normalmente introdotta dalla congiunzione SE. In un'ipotesi possibile o irreale, la frase-condizione può presentarsi anche senza SE: fossi in te , non lo farei = (se fossi in te , non lo farei ) la frase condizione senza SE non è frequenta, e comunque è tipica solo dell'italiano parlato. 
frase-condizione di un'ipotesi possibile introdotta da qualora, quando, purché ecc....; ammesso che , posto che, a condizione che . Oltre che da se, la frase-condizione della sola ipotesi possibile può essere introdotta dalle espressioni: ammesso che, concesso che, posto che, a condizione che, a patto che, nell'ipotesi che, nell'eventualità che :farò qualsiasi cosa, a patto che tu venga dopo tutte queste congiunzioni ed espressioni, si adoperano il congiuntivo presente se la condizione riguarda il presente - futuro e il congiuntivo passato se la condizione riguarda il passato: te lo farò sapere, purchè tu non lo dica a nessuno (condizione nel presente (futuro ),nel caso lei abbia già pagato , non tenga conto di questo lettera (condizione nel passato ) . La condizione può essere espressa con un gerundio, un participio passato (da solo preceduta dalla preposizione A: -lavorando di meno (= se lavorassi di meno )non saresti così stanco . -amministrata meglio (=se fosse amministrata meglio), la città sarebbe più vivibile . -a sentirlo parlare (=se lo si sente parlare), sembra che i problemi li abbia solo lui. Ipotesi con condizione o conseguenza sottintese . In alcuni casi nella lingua parlata in particolare, possono essere sottintesa o la condizione o la conseguenza : Esempi di condizione sottintesa :scusatemi, vorrei con molto piacere, ma stasera devo lavorare (=vorrei con molto piacere, se potessi ; ma ...) ha avuto il coraggio di dire queste cose in pubblico ? se ci fossi stato Io .... (=se ci fossi stato Io non le avrebbe detto; oppure glielo avrei impedito ecc....) frase-condizione senza verbo espresso. Qualche volta, nella frasecondizione è sottinteso il verbo essere: se possibile, vorrei comprare il biglietto prima di venerdì (=se fosse possibile ...) Acquistiamo autovetture di qualunque marca a modello purchè in buone condizioni (=purchè siano in buone condizioni) .

Adesso leghiamo questa parte teorica con quella applicata confrontando la frase condizionale ipotetica con quella araba. 


\section{parte applicata}

\section{Punti di corrispondenza:}

La frase condizionale in arabo ha la stessa struttura di quella italiana perchè come dice Agnese ${ }^{7}$ è costituita da tre parti:

La particella della condizione اداة الثرط

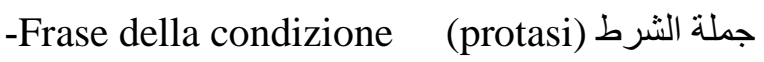

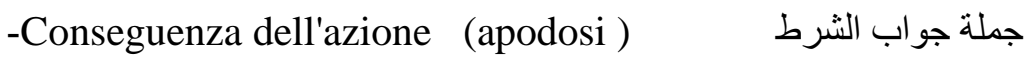

le due proposizioni: una enuncia una ipotesi o una condizione e l'altra ne indica la conseguenza facendo da risposta a quanto enunciato dalla prima. Questo tipo di proposizione è detta anche "frase doppia "

1-Se l'ipotetica è irreale o irrealizzabile:

a) La condizione الثرط viene introdotta dalle particelle: لو "لو لم "se" لو "se non ",seguite da un verbo ; oppure da لو لو لو "se non " (seguite da un nome ).

b) La proposizione جواب viene introdotta da una J rafforzativa (non traducibile) che si omette solo davanti alle particelle negative, ad eccezione di ما ل.

c)Il verbo in entrambe le proposizioni è un "perfetto" per es.,

$$
\text { لو قبل دعوتنا لكان ضيفنا }
$$

Se avesse accettato il nostro invito, sarebbe stato nostro ospite.

$$
\text { لو درسنا لما رسبنا في الإمتحانات }
$$

Se avessimo studiato non saremmo stati bocciati agli esami.

$$
\text { لو لم يكن طبيبا لما استطاع أن يساعدنا }
$$

Se non fosse stato un medico, non ci avrebbe potuto aiutare.

$$
\text { لو لا المدارس لكنا من الجهلاء }
$$

Se non ci fossero le scuole, saremmo degli ignoranti.

2)Se l'ipotetica è reale o possibile

1Cfr., Manca Agnese, Grammatica (teorico-pratica)di Arabo Letterario, A.N.D.A., Roma 1989, pp.314-315. 


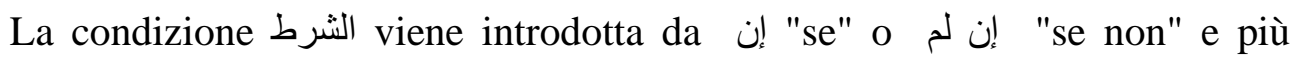

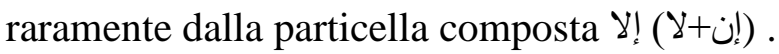

Il verbo, in entrambe le proposizioni, può essere un "perfetto" o uno" iussivo", con la differenza che :

a) Ad uno iussivo nella condizionale deve corrispondere uno iussivo nella إن تسمح نبق عندك es., Se permetti noi restiamo da te جواب proposizione

b) Ad un perfetto nella condizionale, corrisponde un perfetto nella risposta .

Una ف disgiuntiva viene messa all'inizio della risposta se questa non è introdotta da un perfetto o da uno iussivo retto da لم . La suddetta ف viene, tuttavia, omessa se la proposizione di risposta o proposizione consequenziale è antecedente alla condizione stessa .

Esempi:

Se parti parto con te إن سافرت سافرت معك

سوف أغادر المدينة إن كنت ترغب في ذلك Lascerò la città se desideri questo

Se non permetti non resteremo da loro إن لم تسمح فلن نبقى عندهم Se la considera e le vuole bene non la ripudierà mai

$$
\text { إن قدر ها و أحبها فلن يطلقها أبدا }
$$

إذا può essere tradotto sia con "se " che con "quando " perche` indica un ipotesi احتمال

Se il mio amico mi visita andiamo in biblioteca

$$
\text { إذا زارني صديقي ذهبنا إلى المكتبة }
$$

N.B. Se in arabo il tempo è al perfetto non va tradotto al passato in italiano ma secondo il senso.

$$
\text { إذا + ماضي > ماضي }
$$

لو si traduce con "se" e quando un verbo è legato a questa particella e`un verbo che non si realizza e quindi con significato negativo .E` una preposizione non iussiva come إن: non manda all'apocopato e indica sempre un'azione nel passato

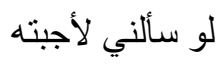

Se mi avesse chiesto gli avrei risposto 


$$
\text { لو + ماضي > ل + ماضي }
$$

N.B. E` possible usare لو إن con le sorelle di كان

$$
\text { " لو " ل " sparisce questo caso la }
$$

$$
\text { لو كان الطالب مجتهدا لنجح }
$$

Se lo studente fosse stato diligente sarebbe stato promesso

$$
\text { لو أن منير ا مسؤول لتركته يسافر لوحده }
$$

Se Mounir fosse responsabile, lo avrei lasciato viaggiare da solo .

إن È una particella che dà il senso di una condizione reale e possibile

$$
\text { إن قبلت الدعوة قبلتها أيضا }
$$

Se tu accetti l'invito, lo accetto / accettero` anche io

$$
\text { لو اجتهدت جيدا لم تتدم }
$$

Se ti fossi impegnato, non ti saresti pentito

$$
\text { إن + إندس > ماضي > ماضي }
$$

Se studi bene, sarai promosso

$$
\text { إن + مجزوم > مجزوم }
$$

لولا E’ una particella del condizionale che significa " se non fosse per" . A differenza di لو لو

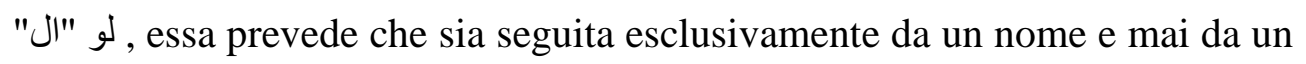
verbo لو لا رحمة اله لضاع الناس لول

Se non fosse per la clemenza di dio, la gente sarebbe persa

$$
\text { لو ال + اسم> ل (+ما ) + ماضي }
$$

Proposizioni ipotetiche introdotte da إن e proposizioni analoghi 
Veccia Vaglieri ${ }^{8}$ ha dato lo schema del periodo ipotetico costituito da una protasi che in arabo "جملة النرط = e da una apodesi introdotta da che

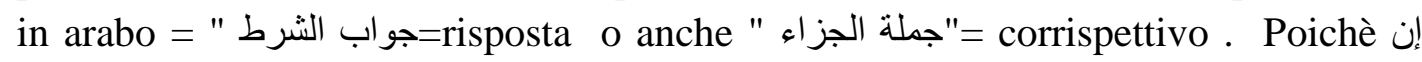
serve ad esprimere una ipotesi reale o possibile, i verbi della protasi e dell'apodosi troveranno corrispondenza o nei loro indicativi (periodo ipotetico della realtà ) o il primo verbo in un loro cong ., il secondo in un loro cond., (il periodo ipotetico della possibilità ). Quanto ai tempi: se l'ipotesi riguarda il presente o il futuro nel caso che si usi l'indicativo, saranno o presenti o futuri ( es., se tu entri, io entro con te إن جئت خرجت معك إلى التنزه , إن دخلت دخلت معك

Se entrerai, io entrerò , se vieni , uscirò con te a passeggio) .

Veccia Vaglier $^{9}$ aggiunge che:

Nel periodo ipotetico, quando la protasi precede (es., se entri , ti picchierà ) si usa :

1) o in entrambe le proposizioni il perfetto ;

2) o in entrambe il condizionale - iussivo;

3) o perfetto nella protasi e il condizionale -iussivo ( o anche l'indicativo ) nell' apodosi;

4) o il condizionale - iussivo nella protasi e il perfetto nell'apodosi.

Se la protasi segue all'apodosi ( es., ti picchierà , se entri ) allora le regole circa il verbo il

riguardano solo la protasi.

Al perfetto o al condizionale - iussivo corrispondono in italiano :

a) nella protasi ( = condizionale ) : l'indicativo presente o futuro ovvero il cong . imperfetto; b)nell'apodosi (= risposta ) : l'indicativo presente o futuro ovvero il condizionale presente .

Quindi il periodo: se fai (o farai o facessi ) questo, muori ( o morirai o morresti ) sonerebbe in arabo

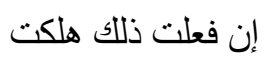

1Cfr., Veccia Vaglieri, Grammatica teorico-pratica della Lingua Araba, vol. II, Istituto per l'oriente, Roma 1989, p.229.

1lvi, Vol., l, p.131. 


\section{إن تفعل ذللك تهـلك \\ إن فعلت ذللك تهلك \\ إن تفعل ذلك هلكت}

Il modo condizionale - iussivo è di norma quando la proposizione o le proposizioni siano negative; allora la negazione è

Questo tipo che è "proposizioni ipotetiche introdotte da" corrisponde al terzo caso della frase condizionale in italiano

Cioè il caso "della irrealità" o, meglio, "della impossibilità" es., ( "se fosse stato qui , avrebbe combattuto anch' egli " ; "se avesse vissuto , avrebbe ora settant' anni ")

Quanto ai verbi ${ }^{10}$,essi vanno:

1)entrambi al perfetto ; es.,se il tuo Signore avesse voluto (o volesse ), avrebbe fatto ( o farebbe ) dell'umanità un popolo unico (Cor.II ,120 ) imprigionato .

لو كنت هنا لما حبس أخي

Quando sono premessi due كان ad entrambi i perfetti, essendo in tal modo trasportata

l'ipotesi nel passato, si userà nella traduzione italiana il trapassato del congiuntivo nella protasi e il passato del condizionale nell'apodosi ( es.,se lo avesse trovato [ in passato], lo avrebbe ucciso

$$
\text { لو كان وجده لكان قتله }
$$

Potrebbe anche aversi un solol avanti al perfetto della protasi ; allora soltanto l'ipotesi riguarderebbe il passato ; es.,

se egli fosse arrivato, te ne avvertirei.

2) Il verbo della protasi all'imperfetto indicativo e quello della apodosi al perfetto; allora in italiano corrisponderà al primo verbo l'imperfetto congiuntivo e al secondo verbo il condizionale presente es.,

لو نشاء حبسناهم

1lvi, Vol., II, pp. 237-238. 
3) All'imperfetto indicativo della protasi può essere premesso un كان allora ,traducendo in italiano , si potrà usare anche il trapassato del congiuntivo e il passato del condizionale rispettivamente nella protasi e nell'apodosi, es.,

se avesse saputo che eri bisognoso, ti avrebbe aiutato

Accanto al semplice g troviamo:

a) lett. Se fosse che, quando si voglia far seguir

una proposizione nominale invece di una verbale; es., se noi avessimo Saputo ciò , non saremmo partiti ; و أنا علمنا بذلك لما سافرنا

b) Spesso senza verbo,nel qual caso corrisponde all'italiano"se" non fosse per ovvero " se non fosse stato per" ovvero " se non ci fosse stato"; لو لا علي لهلك عمرو se non fosse stato per Ali = $($ o se non ci fosse stato Ali $)$, Amr sarebbe perito .

Se a لو لو segue un pronome, questo si congiunge a $\gamma$ nella forma del pronome suffisso ; es., لو لاي للهكت se non fosse stato per me, saresti perito .

Apodosi sottintesa : L'apodosi di una protasi da إن può mancare , la suggerisce allora il contesto, tanto più che spesso la frase successiva incomincia con un إن إن شهد للك عدلان من المسلمين و إلا فاستوف منه اليمين altrimenti ; es., due musulmani ineccepibili testimoniano in tuo favore, [bene]; altrimenti esigi da lui il giuramento .

Proposizioni ipotetiche introdotte da إذا

$V_{\text {eccia }}{ }^{11}$ continua dicendo che Si identificano con le temporali introdotte da questa stessa congiunzione ; è da rilevare innanzi tutto che إذا ha implicito un senso ipotetico, sicchè corrisponde forse più ad un " quando " puramente temporale, e spesso ad un " se " in italiano

إذا Infatti segue regole sue proprie e cioè:

1)è seguita di norma dal perfetto e se la proposizione è negativa da لم e il condizionale- iussivo, ma questo perfetto e questo condizionale - iussivo hanno il valore dell'imperfetto arabo ; perciò vanno tradotti in italiano e col presente o col

${ }^{1}$ Ivi, vol., II, p.240. 
futuro o, il contesto riguardando il passato, con l'imperfetto o ancora, se prevalga in il senso ipotetico, con l'imperfetto congiuntivo ; in arabo si ha il perfetto perchè l'azione è considerata come compiuta analogamente a quanto avviene nelle ipotesi.

\section{Ipotesi riguardanti il passato}

$V_{\text {eccia }}{ }^{12}$ dice che poichè il perfetto nel periodo ipotetico arabo esaurisce la sua funzione presentando come fatti compiuti quelli esposti nella protasi e nell'apodosi, come si esprime in arabo una ipotesi riguardante il passato :

Bisogna distinguere : 1)soltanto la protasi riguarda il passato e l'apodosi il presente ( es."se avessimo acquistato case in quell'epoca , oggi saremmo ricchi "); 2) sia protasi che apodosi riguardano il passato ; in questo caso in italiano si usa l'indicativo per la supposizione probabilmente rispondente a realtà ("se hai detto questo, hai sbagliato "), il trapassato congiuntivo nella protasi ( col passato del condizionale nell' apodosi ) per la supposizione che non ha avuto rispondenza nella realtà , ma poteva averla , e pertanto ad essa corrisponde in arabo il tipo di proposizione ipotetica che è introdotto da إن

${ }^{1}$ Ivi, vol., II, pp.234-235. 


\section{Problemi della traduzione della frase condizionale}

Il traduttore ha tradotto la frase condizionale ognuna secondo il senso e il contesto della frase: il genitivo come in:

1) Quبه الجملة

Con tutta probabilità i calcoli del mio inseguitore tendono invece a prevedere se riuscirà a passare il semaforo con la stessa mia ondata, se riuscirà a tenermi dietro finchè le auto che ci separano, si saranno disperse in varie direzioni . (l'inseguimento, p.105)

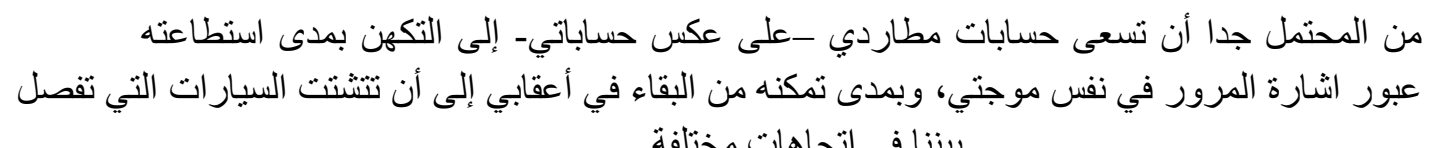

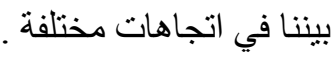

2) In questo esempio " se " è venuta con valore interrogativo perciò il traduttore l'ha tradotto con il problema insomma è se la colonna è divisibile in una serie di segmenti dotati ognuno di vita propria o se la si deve considerare come un corpo unico ed inscindibile, in cui il solo cambiamento che ci si può aspettare è il decrescere della densità con le ore della notte, fino a un punto limite di rarefazione in cui solamente le nostre due auto conserveranno la stessa direzione e tenderanno ad annullare la distanza . (p.105)

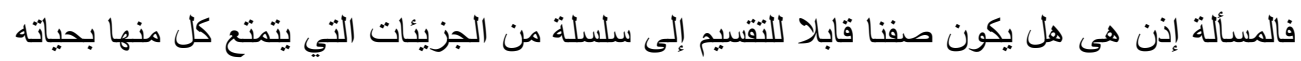

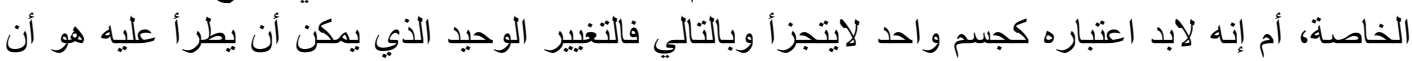

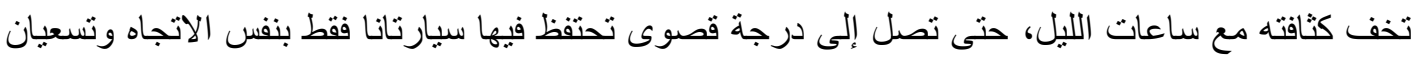
إلى إلغاء المسافة بينهما .

3) Qui il traduttore ha tradotto il secondo caso della frase condizionale (caso della possibilità) con إن+الفعل الماضي في جملة فعل الثرطو الفاء +سوف +الفعل المضارع في جملة

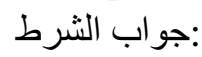

Per uscire da questa situazione il sistema più semplice sarebbe uscire dalla macchina, se uno di noi o tutti e due lasciassimo le nostre auto e proseguissimo a piedi, ritornerebbe a esistere uno spazio e la possibilità di muoverci nello spazio . (p.106) 
قد تكون أبسط وسيلة للخروج من هذا الموقف هي الخروج من السيارة، فإن ترك أحدنا أو نركنا

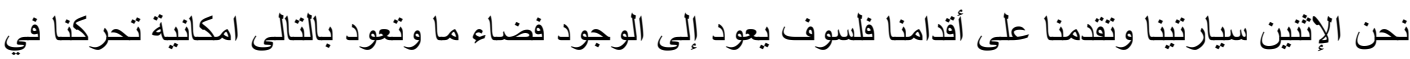
الفضاء.

4) Il traduttore ha tradotto "se" nella frase di "protasi " con متى +إن + الفعل e la frase di " conseguenza cioè apodosi " con أنماضي الهاء + الفعل المضارع come in:

é chiaro che non devo uscire dalla mia macchina neanche se lui abbandona la sua. (p. 104)

$$
\text { من الو اضح إذن أنه يجب على ألاأخرج من سيارتي حتى إن ترك هو سيارته . }
$$

5) Il traduttore ha tradotto il caso dell'impossibilità con الفنب السين +انعل nella frase di conseguenza " apodosi " e con il condizionale -iussivo nella

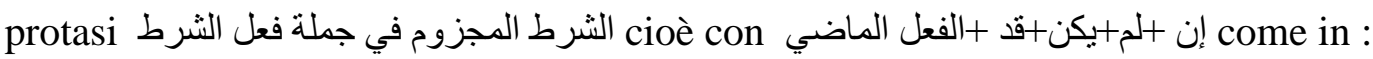

Certo l'avvrebbero rincorso e riportato con la forza a chinare il capo sul volante, se egli non si fosse affrettato a riprendere il suo posto . (p. 106) كانو ا بكل تأكيد سيلاحقونه ويعيدونه بالقوة لينكس رأسه فوق عجلة القيادة، هذا إن لم يكن قد أسرع

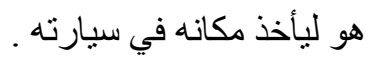

6) Il traduttore ha tradotto il caso dell'incertezza nella protasi con إن + الفعل e nella apodosi con إن + لن+ الفعل المضارع come in:

Il mio inseguitore non oserà mai raggiungermi a piedi perchè anche se facesse in tempo a spararmi non potrebbe poi sfuggire al furore degli altri automobilisti. (p. 106)

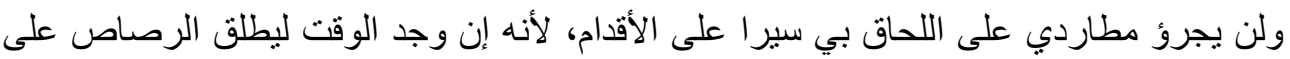

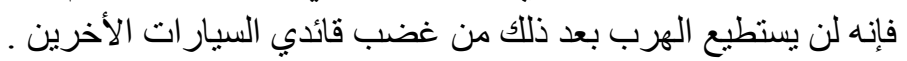

7) Il traduttore ha tradotto il secondo caso della frase condizionale con nella protasi e con إذا +مال الفعل الماضين +الفعل المضارع (nella apodosi come in:

Certo la differenza di velocità tra le varie file sarebbe decisiva se l'inseguitore a un certo punto potesse, avanzando per esempio con la fila di destra , affiancare la sua vettura alla mia ...(p.107)

من المؤكد أن فارق السر عة بين مختلف الصفوف سيكون حاسما إذا ما استطاع مطاردي أن يتقدم -

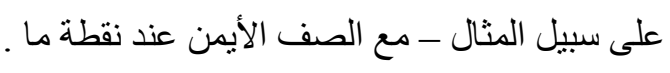


8) Il traduttore ha tradotto la protasi nel secondo caso cioè della possibilità con عندئذ + إذفعل المضارع e l'apodosi con secondo il contesto come in:

Se però il numero di queste macchine - intervallo crescesse o diminuisse, allora il nostro inseguimento tornerebbe a essere un vero inseguimento, indipendentemente dalle nostre velocità o libertà di movimenti.(p.109)

أما إذا زاد عدد هذه السيار ات الفاصلة أو نقص فعندئذ تعود مطاردتنا لتصبر مطاردة حقة، بغض

$$
\text { النظر عن سرعتينا أو حرية حركتنا. }
$$

9) Il traduttore ha tradotto il caso dell'incertezza con السين+ فعل ماضي nella protasi e con كأنني + فعل ماضي nell'apodosi come in:

Basterebbe che alcune di queste macchine affluenti si inserissero tra me e lui, e subito il mio distacco aumenterebbe, cioè sarebbe come se io fossi scattato in una fuga improvvisa .

يكفي أن تدخل بيني وبينه بعض هذه السيارات المتقاطرة، وللتو سيزداد الفاصل بيننا، وسأصبح وكأنني اندفعت في هرب مفاجئ.

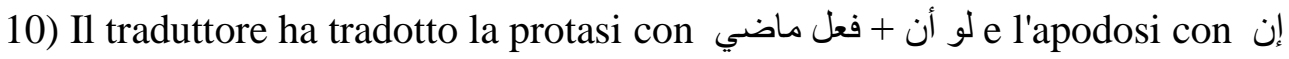
come in:

Invece alla nostra sinistra, in mezzo alla via, ora comincia una stretta isola adibita a parcheggio; se ci sono o si

creano dei posti liberi basterebbe che alcune delle macchine - intervallo decidessero di parcheggiare ed ecco il mio inseguitore vedrebbe tutt'a un tratto accorciarsi la distanza che ci separa. (p.109)

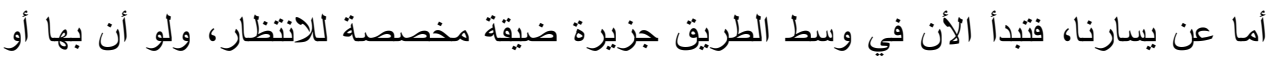

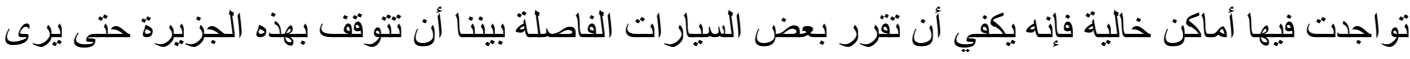

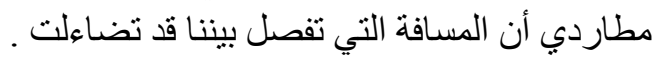

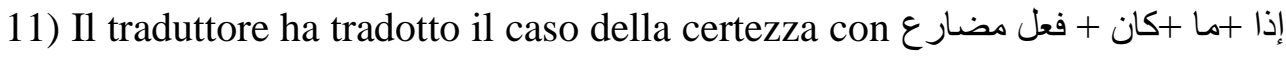
nella protasi e con الفاء + الفعل المضار nell'apodosi come in:

Se quest'affermazione sembra in contrasto con l'esperienza tanto mia che del mio inseguitore, è perché si tratta d'una proprietà non dei singoli corpi ma di tutto l'insieme dei corpi nelle loro relazioni reciproche ...(p.110)

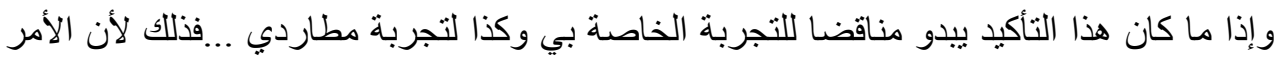
لايتعلق بإحدى خصائص الأجسام بصفتها المنفردة وإنما هو ينعلق بخصائص ولئص الأجسام ككل في علاقاتها 
12) Il traduttore ha tradotto la protasi con إذا +ما +كان+ صفا e con فعل nell'apodosi come in:

Se ogni macchina -fermo restando il senso di marcia il senso d'inseguimento - equivale a ogni altra macchina, le proprietà d'una qualsiasi macchina possono essere attribuite anche alle altre. (p.111)

إذا ما كانت كل سيارة - طالما أن اتجاه الحركة و اتجاه المطاردة ثابتان - مساوية لكل سبارة أخرى،

$$
\text { فان خصائص أي سيارة يمكن إسنادها إلى الأخريات . }
$$

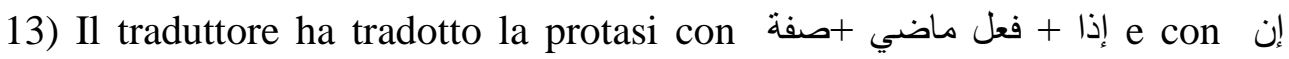
الضمير هاء +فعل المضارع nell'apodosi come in :

A pensarci bene, se tutte le macchine sono coinvolte in inseguimenti, bisognerebbe che la proprietà inseguitrice fosse commutativa . (p.112) و إذا ما أمعنا التفكير فإنه إذا كانت كل السيارات منورطة في مطاردات، فإنه ينبغي أن تكون خاصية

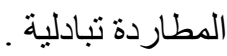

14) Il traduttore ha tradotto la protasi nel caso dell' incertezza con + إذأ e nell'apodosi con الفاء + إن + يكون +قد+ فعل ماضي ماضي come in :

Ma se due secondi prima che ciò avvenga l'inseguitore del mio inseguitore venisse raggiunto e ucciso dal suo inseguitore, il mio inseguitore sarebbe salvo e libero di uccidermi. (p.113)

أما إذا ما حدث قبل هذا بثانيتين أن لحق بمطارد مطاردي مطارد أخر فقتله، فإن مطاردي يكون قد

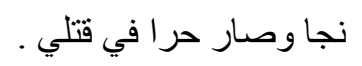

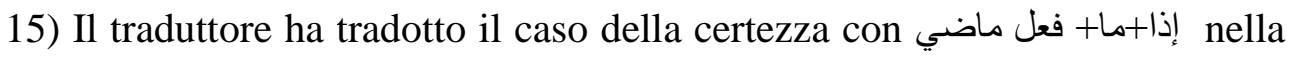
protasi e con الفاء+لا +فعل مضارع nell'apodosi come in :

Ma se ammetto l'esistenza d'una catena d'inseguimenti dietro di me, non c'è ragione che questa catena non si prolunghi anche attraverso di me nella parte della colonna che mi precede . (p.113)

لكن إذا ما سلمت بوجود سلسلة من المطاردات خلفي فلا يوجد ما ييرر عدم امتداد هذه السلسلة أيضا عبري لتشمل ذللك الجانب من الفرقة الذي يسبقني .

16) In questa frase, la protasi è tradotta con إسم e l'apodosi con إذاب فعل ماضي إسم موصول + فعل مضارع come in :

Cioè la sola alternativa che conta è se la mia condizione d'inseguito è destinata a rimanere terminale e asimmetrica o se anch'io sono a mia volta un inseguitore. (p.113) 
أي أن البديل الوحيد الذي يعتد به هو إذا كانت حالتي كمطارد أن تكون حالة نهائية غير منسقة مع

$$
\text { غير ها أو أن كنت أنا بدوري مطارد . }
$$

17) In questa frase, la protasi è tradotta con إذاب+ ما +كان +فعل مضارع e أن+ فعل مضارع nell'apodosi con

Come nella prima frase e con إذاب كان + إناك nella protasi e nell'apodosi con إن +فعل مضارع come nella seconda frase in questo paragrafo:

Per sapere se questa ipotesi corrisponde al vero, non ho che da allungare la mano : se sul portaoggetti della mia macchina c'è una pistola pistola, è segno che sono anch' io un inseguitore . (p.114)

لكي أعرف إذا ما كان هذا الافتراض يتطابق ويتمشى مع الواقع، ليس على إلا أن أمد يدي : فإذا

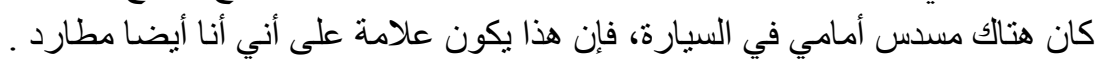

18) In questa frase la protasi è tradotta con إن + كان + فعل مضارع e nell'apodosi con الفاء + الفعل المضارع come in :

La macchina che mi precede si trova in una brutta posizione, avendo superato la linea del semaforo

Il guidatore si volta per vedere se può fare marcia indietro, mi vede, ha un' espressione di terrore (p.114)

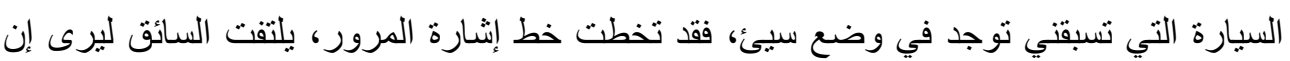

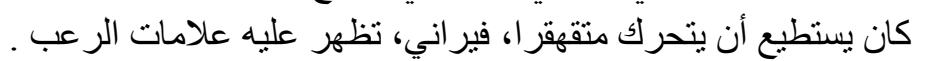




\section{Conclusione}

Ho fatto questa ricerca spinta da una curiosità e da una voglia di sapere di più delle caratteristiche e delle funzioni della frase-condizonale nella lingua italiana e quella araba. Sono giunta alla conclusione che il condizionale è un elemento fondamentale nella formazione della lingua e che le sue origini ritornano alla lingua latina. In questa parte della ricerca, ho osservato il condizionale ed ho cercato di verificare la verità delle ipotesi riguardanti il condizionale. Ricapitolando, il condizionale presente può svolgere diverse funzioni, esso può avere valore di esprimere un desiderio, chiedere o dire qualcosa in modo cortese ,dare consigli ,esprimere dubbio,presentare una notizia come non certa nel presente, esprimere un'azione che dipende da un'altra, anche il condizionale passato ha il valore di esprimere un'azione che non si è potuta realizzare nel passato o che non si può realizzare nel presente o nel futuro, esprimere un'azione futura rispetto ad un momento del passato ,presentare una notizia come non certa nel passato . Alla fine, vorrei parlare dei punti di corrispondenza e problemi di traduzione. Ho notato che: la frase condizionale in arabo ha la stessa struttura di quella italiana. Se in arabo il tempo è al perfetto non va tradotto al passato in italiano ma secondo il senso. Le proposizioni ipotetiche introdotte da "لو corrispondono il caso dell'impossibilità della f. cond. in italiano. Le proposizioni ipotetiche introdotte da "إن" corrispondono il caso della realtà e della possibilità della $\mathrm{f}$. Cond., in italiano . Se prevalga in" إذا" il senso ipotetico, con l'imperfetto congiuntivo ; in arabo si ha il perfetto perchè l'azione è considerata come compiuta analogamente a quanto avviene nelle ipotesi . Nelle ipotesi riguardanti il passato, sia protasi che apodosi riguardano il passato ; in questo caso in italiano si usa l'indicativo per la supposizione che non ha avuto rispondenza nella realtà , ma poteva averla, e pertanto ad. essa corrisponde in arabo il tipo di prop. ipotetica che è introdotto da "إن" . Quando traduciamo la f. cond. dall'italiano in arabo, è tradotta ognuna secondo il senso della frase e secondo il contesto come in :

- Quando traduciamo il caso della realtà, possiamo tradurlo con شبه الجملة il genitivo.

-Qualche volta " se " viene con valore interrogativo perciò si traduce con هل

- il traduttore ha tradotto il secondo caso della frase condizionale (caso della possibilità) con إن+الفعل الماضي في جملة فعل الثرطو الفاء +سوف +الفعل المضار ع في جملة

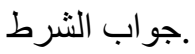

حتى +إن + إن أن la frase di " protasi" con الفعل الماضي الهاء + الفعل المضارع 
-Il traduttore ha tradotto il caso dell'impossibilità con كان+ السين +الفعل المضارع nella

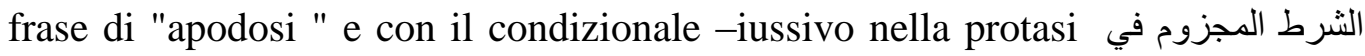

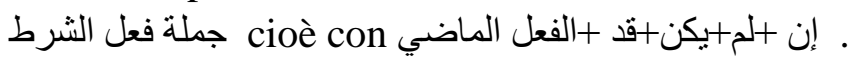

-Il traduttore ha tradotto il caso dell'incertezza nella protasi con إن + الفعل الماضي e nell'apodosi con إن + لن+ الفعل المضارع .

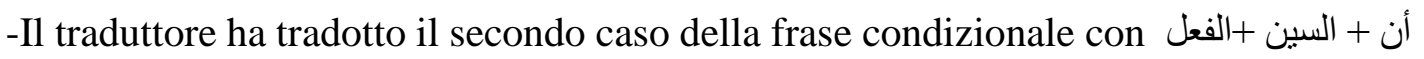
nella protasi e con إذا +مال الفعل الماضي عell'apodosi.

-Il traduttore ha tradotto la protasi nel secondo caso cioè della possibilità con + إذا e l'apodosi con عندئذ + الفعل المضل الماضي cioè secondo il contesto.

-Il traduttore ha tradotto il caso dell'incertezza con السين+ فعل ماضي nella protasi e con كأنني + فعل ماضي nell'apodosi.

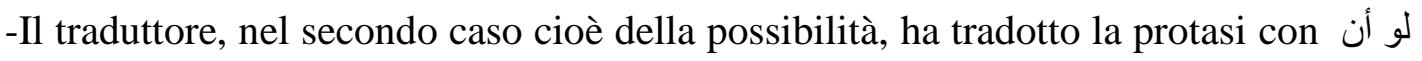
. إن + e l'apodosi con الفعل المضارع + فعل ماضي

-Il traduttore ha tradotto il caso della certezza con إذا +ما +كان + فعل مضارع nella protasi e con الفاء + الفعل المضار nell'apodosi.

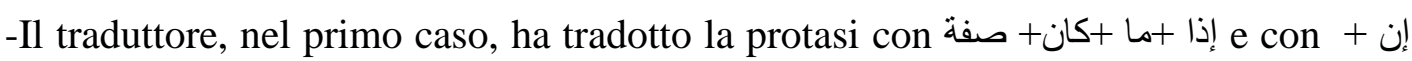
فعل مضارع nell'apodosi.

-Il traduttore, nel secondo caso, ha tradotto la protasi con إذا + فعل ماضي +صفي e con إن +الضمير هاء +فعل المضار ع nell'apodosi.

-Il traduttore ha tradotto la protasi nel caso dell'incertezza con إذا + أن + فعل ماضي e nell'apodosi con الفاء + إن + يكون +قد+ فعل ماضي ill' .

-Il traduttore ha tradotto il caso della certezza con إذابما+ فعل ماضي nella protasi e con الفاء+لا +فعل مضارع nell'apodosi.

إسم e l'apodosi con إذاب+ فعل ماضي ela primo caso anche, la protasi è tradotta con + إسم موصول + فعل مضارع

-Nel primo caso, la protasi è tradotta con إذاب ما +كان +فعل مضار ع e nell'apodosi con إذاب كان +هناك nella protasi e nell'apodosi con

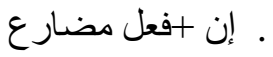

-Qualche volta, la protasi è tradotta con إن + كان + فعل مضار e nell'apodosi con إن إعل nel primo caso.

Concludendo, sperei di essere riuscita a rappresentare l'argomento e ad esplorarlo in modo sufficiente. 


\section{Bibliografia}

-Agnese, M., Grammatica (teorico-pratica) di Arabo Letterario,A.N.D.A.,Roma,1989.

-Bailini, S., Consonno, S., I verbi italiani, Alma Edizione ,Firenze,2004.

-Battaglia, P.,V., La Grammatica italiana , Zanichelli, Torino, 1980.

- Battista, G., M., L'Italiano come seconda lingua, Milano,1992.

-Battista, G., M., L’italiano come prima e seconda lingua ,Guerra edizione , Perugia,1996.

-Cusatelli, G., Garzanti della lingua italiana, Italia, 1981.

-Dardano, M., e Trifone, P., La Lingua italiana, Bologna, 1985.

-Dardano, M., e Trifone, p., Grammatica italiana con nozioni di linguistica, Bologna, 1995.

-Fogarasi, M., Grammatica italiana del Novecento, Roma, 1969.

- Moretti, M., e Consonni, D., Lingua Madre, Torino, 1978.

-Patota G., Grammatica di riferimento della lingua italiana per stranieri Società Dante Alighieri, le Monnier, Firenze,2003.

-Scarduelli T., e Achiardi, G., Lingua e Grammatica, Milano, 1988.

-Serianni L., La Grammatica Italiana, ,Utet, Urbino, 1989.

- Vaglieri, V., Grammatica Teorico-pratica della lingua araba,vol.,I, II, Istituto per l'Oriente , Roma , 1989.

\section{Sitografia}

http://www.treccani.it/enciclopedia--

-https://www.skuola.net/grammatica-italiana/proposizioni-principalicoordinate-subordinate.html

-http://www.grammatica-italiana.it/proposizioni-subordinate.html

-periodo-ipo...<https://www.ef-italia.it

- Il-p...<www.italianomadrelingua.com

-Period... $<w w w . o n e w o r l d i t a l i a n o . c o m$

-http://affrescodellalinguaitaliana.com 


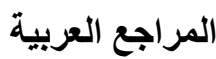

1- ابن عقيل، بهاء الدين، شرح ابن عقيل، ألفية ابن مالك، ج1، ج2، المكتبة التجارية الكبرى، القاهرة، الطبعة الثانية، 1935.

2 - - ابن منظور، لسان العرب، دار المعارف، القاهرة، لم تذكر السنة.

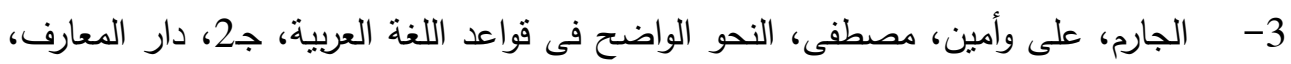

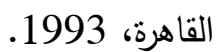

4- - الرازى، أبو بكر، الصحاح، دار نهضة مصر، القاهرة، لم تذكر السنة.

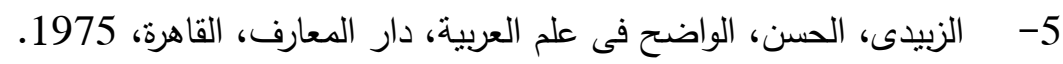

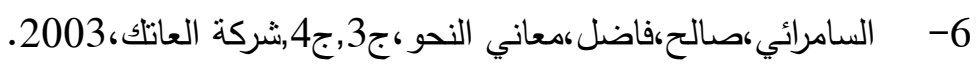

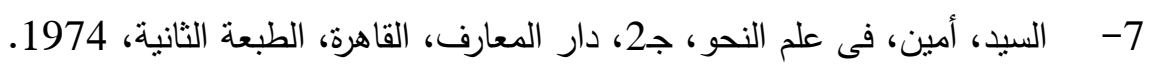

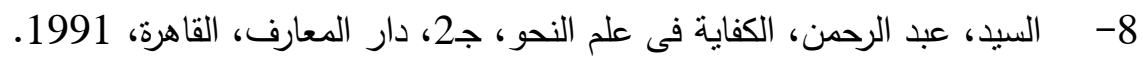

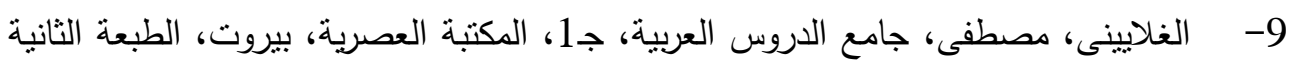
والثلاثون، 1996.

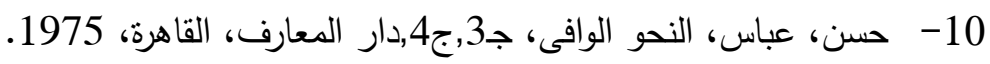
11- عيد، محمد، النحو المصفى، مكتبة الثباب، جامعة القاهرة، 1987 